\title{
Identifying the Nature of Fouling Layers by Online Monitoring of the Propagation of Vibrations Along the Deposition Surface
}

\author{
RENATO TEIXEIRA, $^{1}$ ANA PEREIRA, $^{2}$ JOAQUIM MENDES, ${ }^{\mathbf{3}}$ and LUI'S F. MELO ${ }^{\mathbf{1}}$ \\ $1_{\text {LEPAE, Department of Chemical Engineering, University of Porto, Porto, Portugal }}$ \\ ${ }^{2}$ ENKROTT QUíMICA S.A., Sintra, Portugal \\ ${ }^{3}$ IDMEC-Pólo FEUP, Faculty of Engineering, University of Porto, Porto, Portugal
}

An online fouling monitoring device known as the Mechatronic Surface Sensor (MSS) was applied to a tubular configuration and validated in order to allow a user to identify the nature of the fouling deposits using surface acoustic wave properties, without needing to visualize the fouled surface. This work was based on the formation of simulated milk deposits, with calcium phosphate and protein fouling agents. The results show that by using the system output parameters, such as normalized amplitude and its relationship with the damping factor, it is possible not only to follow the buildup of the deposits, but also to differentiate between the deposits' nature. These parameters showed a clear distinction between a more rigid deposit (e.g., simulated milk ultrafiltrate [SMUF]) and more viscoelastic ones (e.g., WPI or a mixture of SMUF with WPI).

\section{INTRODUCTION}

Fouling is an unwanted, undesirable, but unavoidable phenomenon. In industrial situations it has a major impact on various types of equipment, but primary concerns relate normally to heat transfer equipment [1]. In the oil industry, for example, fouling translates to major efficiency losses in the heat exchanger network (HEN). These losses represent a lower in- let crude temperature at the furnace, thus requiring additional costs in terms of fuel usage for the furnace to provide the in- tended crude temperature for an optimal distillation operation [2]. Applications that require water as a cooling agent can be afflicted with a specific type of fouling, such as the formation of biofilms and the deposition of other organic or inorganic matter [3, 4]. Regarding processing industries, one of the major affected areas is in food processing plants, such as dairy producers. According to a number of previous studies [5, 6], milk production is often plagued with fouling from two origins, "mineral deposition and protein denaturation." While the mineral deposition occurs mainly due to calcium phosphate derivatives, amorphous calcium phosphate (ACP, $\mathrm{Ca} 3(\mathrm{PO} 4) 2 x \mathrm{H} 2 \mathrm{O}$ ), dicalcium phosphate dihydrate (DCPD, CaHPO4 $2 \mathrm{H} 2 \mathrm{O}$ ), octa- calcium phosphate (OCP, $\mathrm{Ca} 8 \mathrm{H} 2(\mathrm{PO} 4) 65 \mathrm{H} 2 \mathrm{O})$, and hydroxyapatite (HAP, $\mathrm{Ca} 5(\mathrm{PO} 4) 3 \mathrm{OH})[7,8]$, the denaturation of proteins by heat such as $\alpha$-lactalbumine and especially $\beta$-lactoglobulin [9] is also responsible for the fouling taking place. 
Attacking the source of the problem has had many different approaches, such as improving heat exchanger design [10], using antifouling chemical agents or antifouling surfaces [11, 12], or optimizing production-cleaning cycles with the help of online noninvasive monitoring systems [13, 14].

Online fouling monitoring is usually carried out in industrial settings by assessing heat transfer parameters (decrease in the overall heat transfer coefficient over time), or measuring pressure drop variations as the fouling layer develops. Additionally, new methods have been developed in the last decade, some of which are not yet fully implemented in production plants [15]. One important issue for operators is to be able to have some knowledge of the nature of deposit that is building up in the heat exchanger, in order to choose the best option for the preventive treatment or for the cleaning process/chemicals to be applied in each situation. For example, in cooling-water systems, de- pending on the water biochemical properties, the deposit may be predominantly formed by precipitation of inorganic salts and adhesion of small suspended inorganic particles (clay, silt, iron oxides), by biofilm growth, or by a mixture of different types of fouling agents. In milk heating processes, either inorganic (calcium-based) or organic (protein) deposits or both may be formed $[6,16]$.

Therefore, the online monitoring system should ideally be able to not only follow the growth and detachment of the de- posits, but also give some information on the generic nature of the fouling layer.

This work demonstrates the use of a sensor based upon the analysis of vibration properties of acoustic waves along a fouled surface and shows how the type of deposit can be identified by analyzing only the vibration data processed in the device. The technology is known as the Mechatronic Surface Sensor (MSS). Taking on previous work undertaken by Pereira et al. [17-19] with a semicircular cross-section laboratorial flow cell, the MSS technology was adapted to a tubular configuration by Enkrott Química and LEPAE, as described in the Materials and Methods section that follows. Relying on the sensor's online and real-time measurements capabilities, deliberate fouling experiments were performed to monitor, in a nonintrusive way, what was happening on the inner surface of the tube. The work shown in this paper was conducted in order to validate this new configuration, and to allow further identification of the type of deposit formed using the output response of the system. As an illustrative example, the case of (simulated) milk deposits was studied, including calcium phosphate and protein fouling agents.

\section{MATERIALS AND METHODS}

\section{Materials}

The experimental assays focused on the analysis of the formation of deposits with different characteristics.

A simulated milk ultrafiltrate (SMUF) solution was used to form calcium phosphate deposits in nature, prepared according to the formulation in Jenness and Koops [20]. It consists of an aqueous solution that models the mineral composition of milk, which was kept overnight at $5{ }^{\circ} \mathrm{C}$ before each experiment took place. When added to the recirculating tank in each assay, the $\mathrm{pH}$ value was adjusted to 6.80 .

The second type of intended deposit was of an organic nature, based upon a whey protein isolate (WPI, Laprodan DI-9224, supplied by ARLA Foods, Denmark). The composition was $88 \%$ 
protein $(\mathrm{w} / \mathrm{w})$, being 15\% $\alpha$-lactalbumin ( $\alpha-\mathrm{La}), \quad 55 \% \quad \beta$ - lactoglobulin $(\beta-\mathrm{Lg}), 20 \%$ glycomacropeptide, and lesser quantities of immunoglobulin $\mathrm{G}$, bovine serum albumin, lactose, fat, ash, minerals, and moisture.

A phosphate-buffered saline (PBS) solution was used for the WPI dissolution, in order to obtain $3 \mathrm{~g} / \mathrm{L}$ of $\beta-\mathrm{Lg}$, as done in similar previously performed work by Santos et al. [21].

For the tests with a blend of SMUF and WPI, the assays started off with the addition of SMUF to the system, as described earlier, and then after initial stabilization, a small part of the circulating fluid was removed for the WPI dissolution. After obtaining a good homogenization, this mixture was added to the tank for the continuation of the assay.

For cleaning procedures between assays, nitric acid $65 \%(\mathrm{w} / \mathrm{w})$, of analytical grade, was used after the SMUF experiments and sodium hydroxide $2 M$, analytical grade, for the WPI ones. In each case, after this step an alkaline detergent, RBS- 35 (Chemical Products, R. Borghgraef s.a., Belgium) was used for additional cleaning, followed by several system rinses with water. All salts used were of analytical grade.

\section{Experimental Apparatus}

The assays were conducted in a test rig similar to the one shown in Figure 1. It consists of a jacketed tank where the aqueous solution is placed (deionized water or a PBS solution) and the chemicals are added. All of the tank's surfaces in contact with the liquids are made of AISI 304 stainless steel. The tank has a temperature controller and recirculation system for homogenization and is connected to a circulation pump, which in turn is connected to a piping system. The piping system is made up of vertical parallel stainless-steel pipes, SS AISI 304, of equal diameters, di 14 $\mathrm{mm}$, where one pipe has the MSS mounted on it and another pipe is the coupon line (which comprises six coupon elements similar to the one shown in Figure 1). The monitored zone of the sensor line, where the MSS is placed, is aligned with the coupons, at the same level height. This means that the coupon line is mounted in such a way as to guarantee that the deposition occurring on the coupons happens in the same zone as the deposits forming on the monitored surface zone of the sensor line. Furthermore, preliminary experiments were carried out in order to evaluate the amount of mass on each coupon of the coupon line. Those results indicated similar dry masses for the coupons independently of their position within the coupon line. The coupons are made of the same material, SS AISI 304, and are $60 \mathrm{~mm}$ in length. They have a semicircular cut on one of the extremities, $10 \mathrm{~mm}$ in length, to allow them to be mounted in pairs, and when dismounted also enable deposit thickness determination (an enlarged example of a coupon can be seen in Figure 1). Each coupon was weighed before the beginning of the fouling assay while clean and dry. Furthermore, each coupon was weighed immediately after its removal (subtracted by the initial weight gives the wet mass determination), and after achieving constant mass in an oven at $105^{\circ} \mathrm{C}$ (subtracting the initial weight provides the dry mass determination). The thickness is determined while the deposit is still wet, utilizing a digital micrometer, equivalent to the procedure used in Brito and Melo [22].

The MSS technology comprises two distinct elements placed on the outer surface of the pipe: an actuator, a piezoelectric ceramic bender (BM 70/25/200M, from Piezomechanik, GmbH, Germany), and a sensor, an accelerometer chip (ADXL-103, from Analog Devices, USA). The concept of the MSS "relies on the fact that the attachment, growth and detachment of deposits produce changes on the vibration properties of surface waves" [18]. The already-mentioned 
actuator makes the tube vibrate according to a specified chosen frequency, which is one of the system's resonant frequencies (the one with highest magnitude). It transforms an electrical excitation, generated via a data acquisition board (National Instruments) connected to a computer, into a mechanical impact. Consequently, a full yield response is obtained; the produced vibration is propagated along the surface and received by the accelerometer, which is also connected to the data acquisition system. Each one of these produced waves is acquired at specific user-defined intervals. The data acquisition system then processes mathematically the acquired waves to determine the highest amplitude after fast Fourier transforms (FFT). It should be stressed that the amplitude of the vibrations is of the order of nanometers, which does not affect the structural properties of the surface material. For further detailed information on the technical specifications refer to previous published work by Pereira et al. [17-19].

Other elements were mounted as well, such as a control valve for flow regulation and a flowmeter for flow measurement at the beginning of each pipe, and a temperature sensor at the end. These two last elements were connected to the data acquisition system for online and real-time flow and temperature measurements. The piping system is then connected back to the initial tank, closing the fluid circuit.

\section{Fouling Experiments}

All assays were performed with equal hydrodynamic properties, such as a temperature of $60^{\circ} \mathrm{C}$ and equal flow velocities of $0.75 \mathrm{~m} / \mathrm{s}$ in all pipes, thus guaranteeing similar Reynolds numbers, of values around 19,000, and subsequently obtaining comparable shear stress for each deposit formed.

The temperature was established according to system parameters. For the assays involving WPI the guideline was previous work undertaken by Santos et al. [21], where the data show a higher adsorption of the protein on the SS surface, by using a solution of WPI in PBS, to obtain $3 \mathrm{~g} / \mathrm{L}$ of $\beta-\mathrm{Lg}$, at a $\mathrm{pH}$ value of 6.7 and $85^{\circ} \mathrm{C}$. We replicated these conditions but set the temperature to $60^{\circ} \mathrm{C}$ due to a physical constraint, namely, that a temperature higher than $65^{\circ} \mathrm{C}$ would damage the piezoelectric element. Therefore, the remaining assays performed with the mixture of SMUF and WPI and SMUF alone maintained the same temperature set-point for comparability.

\section{RESULTS AND DISCUSSION}

\section{Monitoring of Fouling Experiments}

The data acquisition system enables the user to receive, on- line and in real time, information on what the sensor is analysing. The data processing of the system's output response includes, among other parameters, the maximum value of amplitude of each produced wave.

The goal of the experiments described in this section is to evaluate the impact of the fouling on the vibrating response of the system, when compared to the one observed for the non- fouled (when the surface is clean) situation. Thus, after adding the fouling agent, each amplitude determined over time $(\mathrm{A} i)$ is subtracted from the amplitude observed for the initially clean surface (A0). This mathematical variable, which enables the comparison of different fouling deposits, was named the normalized amplitude (Anorm) and is represented by Eq. (1): 


$$
\text { Anorm }=\left|A_{i}-A_{0}\right|
$$

Figure 2 shows a graphical representation of the normalized amplitude variation over time. It can be seen that for all the tested deposits (SMUF, WPI, and SMUF WPI) the normalized amplitude increases withtime.

In order to relate this normalized amplitude to the amount of deposit, as in Figure 3, coupons' dry mass values were analyzed at 900 and 3780 minutes of the fouling experiments for each type of deposit.

The quantity (by assessing the dry mass) and structural parameters (by determining the wet density) of the deposit have been evaluated for each sampled coupon (for different sampling times and fouling material). The dry mass variation for the three types of studied deposits can be seen in Figure 3. When analyzing each deposit alone, higher dry masses correspond to higher normalized amplitudes. However, Figure 3 also shows that for similar masses, different deposits have different impacts on the normalized amplitudes. For example, for a dry mass of $0.15 \mathrm{mg} / \mathrm{cm}^{2}$, the normalized amplitude is completely different between the SMUF (Anorm $\sim 0.010 \mathrm{~V}$ ) and the other two deposits (Anorm $0.030 \mathrm{~V}$ ). The comparison between WPI and SMUF+WPI is not possible using this plot, since the experimental data are in different dry mass ranges. However, the trends shown in Figure 3 appear to indicate that if higher masses had been obtained for the WPI deposit, the normalized amplitudes would approach those of the SMUF+WPI fouling layer. Similar results were also obtained by Pereira et al. [18] in the semicircular flow cell configuration.

\section{Structural Characterization of Deposits}

The authors looked for another parameter that could be obtained directly from the MSS measurements and would allow them to characterize the type of deposit being formed on the inner surface of the pipe. This parameter should certainly be related to the so-called damping factor (DF), which is a mea- sure of the energy dissipation along the propagation path. The damping factor can be calculated for each of the sensor's output waves, by using the same methodology as in Pereira et al. [17], according to Eq. (2):

$$
\mathrm{DF}=\frac{\frac{1}{n} \ln \left(\frac{\mathrm{x}_{\mathrm{i}}}{\mathrm{x}_{\mathrm{i}+\mathrm{n}}}\right)}{\sqrt{(2 \pi)^{2}+\frac{1}{\mathrm{n}^{2}} \ln ^{2}\left(\frac{\mathrm{x}_{\mathrm{i}}}{\mathrm{x}_{\mathrm{i}+\mathrm{n}}}\right)}}
$$

where $\mathrm{xi} / \mathrm{xi}+\mathrm{n}$ is the ratio of two nonconsecutive amplitudes, $n$ is an integer, and $\left(\begin{array}{ll}n & 1\end{array}\right)$ is the number of analyzed peaks.

Similarly to the amplitude calculations, variations in the damping factor are obtained by the MSS software for each type of deposit, with DF0 being the damping factor without the foulingagent addition (i.e., initial clean surface) and DFi the value after its addition; see Eq. (3):

$$
\mathrm{DF}_{\text {norm }}=\left|\mathrm{DF}_{i}-\mathrm{DF}_{0}\right|
$$

Contrary to what was found for the flow cell configuration [18], where the preliminary results indicate that the DF seemed to be mainly affected by the structural characteristics of the de- posits, on the tubular geometry, this evidence is not clear. This is related to how the wave propagates through 
the system, and how the deposit characteristics (namely, mass and structure) affect the monitored resonant frequency. Since the flow cell configuration has different physical constraints (O-ring seals, screws, etc.) from the tubular configuration, the wave propagation pattern is also different and, particularly, the amplitude should also be taken into account in the structural analysis. Therefore, the ratio Anorm/DFnorm was considered as a more appropriate parameter to emphasize the structural effect of the deposit on the output wave of the system. Thus, a graphical representation between this Anorm/DFnorm relation and the wet density (an indicator of the deposit compactness) was plotted in Figure 4. The wet density reflects the structural characteristic of the deposit since, as explained in the preceding section (Monitoring of Fouling Experiments), it considers the mass and the wet thickness of the deposits.

An examination+of Figure 4 shows that the SMUF deposit maintains an Anorm/DFnorm ratio value always below $50 \mathrm{~V}$ regardless of the obtained wet density. This seems to indicate that milk deposits of an exclusive mineral/inorganic nature produce lower values of this parameter, whereas the Anorm/DFnorm ratio has a larger variation on the other two types of deposit (more viscoelastic). As seen in an earlier section, the data in Figure 4 confirm that the SMUF WPI mixed layers display vibration properties that are closer to the WPI deposits than to the SMUF inorganic deposits. As a practical conclusion for the cases studied here, an Anorm/DFnorm ratio value above $50 \mathrm{~V}$ indicates that the deposit being formed has a predominantly organic and/or viscoelastic nature.

\section{Functionality Demonstration}

The buildup of viscoelastic deposits (WPI and SMUF+ WPI) has a greater impact on the system's global damping factor than on the system's amplitude (related to the mass). This is explained by the fact that viscoelastic deposits incorporate a larger quantity of water, and hence dissipate more energy/vibration during the wave propagation phenomenon. Therefore, the effect that an equal mass has upon the normalized amplitude is greater as the viscoelasticity of the deposit increases. On the other hand, when considering more rigid deposits with a lower viscoelastic nature (which incorporate less water within their structure), the impact the deposit has on the normalized amplitude (the parameter that monitors/correlates to the quantity of mass) is greater than on structural parameters (like the damping factor).

Thus, as seen in Figure 5a, an Anorm/DFnorm ratio below $50 \mathrm{~V}$ points toward a predominantly rigid deposit. Since the Anorm/DFnorm ratio reflects the impact of the structural change of the deposit in the vibration of the system, relative to its amplitude variation, this indicates that an increase of SMUF dry mass does not change its structural characteristics, establishing an apparent nondependency of SMUF with the Anorm/DFnorm ratio. However, an Anorm/DFnorm ratio above $50 \mathrm{~V}$ indicates a deposit with more (visco)elastic properties (that will greatly increase with a small increase in mass). Furthermore, it seems that when comparing the two viscoelastic deposits (WPI and SMUF+WPI), the Anorm/DFnorm ratio changes within the same range (between $50 \mathrm{~V}$ and $200 \mathrm{~V}$ ), for constant but different Anorm values. The WPI shows an Anorm value of $\sim 0.018 \mathrm{~V}$ and the SMUF+WPI an Anorm value of $\sim 0.038 \mathrm{~V}$. This seems to indicate a possibility to go further in the distinction of fouling layer structures within viscoelastic deposits, although a more thorough experimental analysis is still needed.

In order to confirm the ability to predict the structure of the fouling layer from the output of the MSS, a SMUF deposit was formed and after a given period of time (according to the description of 
the SMUF+WPI assay procedure in the Materials and Methods section) WPI was added. During these experiments, the Anorm versus the Anorm/DFnorm ratio had been determined. Figure $5 \mathrm{~b}$ shows an Anorm/DFnorm value below $50 \mathrm{~V}$ for the SMUF alone, which increased for values between 80 and $200 \mathrm{~V}$ when the WPI was added. These results are in accordance with what was expected, since in Figure 5a the WPI (viscoelastic deposit) also presents an Anorm/DFnorm ratio within the range found for the mixture of SMUF and WPI.

The results shown in this paper demonstrate the ability that the MSS has to follow fouling formation and to provide indication of its structural changes. The industrial implementation of these findings can be quite important on the implementation of proactive measures regarding fouling and cleaning. The information about the fouling structure, in complement to the deposit amount, can enable the optimization of operation and cleaningprocedures.

\section{CONCLUSIONS}

The MSS (Mechatronic Surface Sensor) technology was con- firmed on a tubular configuration, by conducting deliberate fouling experiments to originate three types of deposits: SMUF, WPI and a blend of SMUF and WPI. The data originating from these experiments allowed the establishment of a threshold, using variations in normalized amplitudes and in damping factors. By using this threshold adequately, it is now possible to perform a preliminary identification of certain fouling material characteristics. With the purpose of monitoring a continuous process, it is conceivable to determine an increase in mass (given by the normalized amplitude) and associate it with a known structural behavior.

From an industrial perspective, this understanding of fouling evolution and behavior happening on a surface, without the need to disassemble and visually inspect the system where the fouling phenomenon is taking place, can have significant economic importance in making timely decisions about antifouling measures to be taken and, therefore, in reducing the plant operating costs. Future work to be developed with other deposits will help validate the threshold indicated in this work, as well as others determined a posteriori, and possibly allow us to formulate necessary correlations.

\section{NOMENCLATURE}

A amplitude, $\mathrm{V}$

ACP amorphous calcium phosphate, $\mathrm{Ca} 3(\mathrm{PO} 4) 2 \times \mathrm{H}_{2} \mathrm{O}$

DCPD dicalcium phosphate dehydrate, CaHPO4 2H2O DF damping factor, dimensionless

FFT fast Fourier transforms

HAP hydroxyapatite, $\mathrm{Ca} 5(\mathrm{PO} 4) 3 \mathrm{OH}$ HEN heat exchangernetwork

MSS Mechatronic Surface Sensor

OCP octacalcium phosphate, $\mathrm{Ca} 8 \mathrm{H}_{2}\left(\mathrm{PO}_{4}\right) 65 \mathrm{H}_{2} \mathrm{O}$ PBS phosphate-buffered saline

SMUF simulated milk ultrafiltrate

SS stainless steel

WPI whey protein isolate

\section{Subscripts}

0 at $t=0$, without fouling agent dry after drying procedure

$\mathrm{i} \quad$ at an instant in the assay at $t>0$, with fouling agent present 
norm normalized

wet while wet

\section{REFERENCES}

[1] Bott, T. R., Fouling of Heat Exchangers, Elsevier Science, Oxford, UK, 1995.

[2] Panchal, C. B., and Huangfu, E. P., Effects of Mitigating Fouling on the Energy Efficiency ofCrude-Oil Distillation, Heat Transfer Engineering, vol. 21, no. 3, pp. 3-9, 2000.

[3] O'Toole, G., Kaplan, H. B., and Kolter, R., Biofilm For- mation as Microbial Development, Annual Review of Mi- crobiology, vol. 54, pp. 49-79, 2000.

[4] Melo, L. F., and Bott, T. R., Biofouling in Water Systems, Experimental Thermal and Fluid Science, vol. 14, no. 4, pp. 375-381, 1997.

[5] Lalande, M., Rene, F., and Tissier, J. P., Fouling and Its Control in Heat Exchangers in the Dairy Industry, Biofoul- ing, vol. 1, no. 3, pp. 233-250, 1989.

[6] Bansal, B., and Chen, X. D., A Critical Review of Milk Fouling in Heat Exchangers, Comprehensive Reviews in Food Science and Food Safety, vol. 5, no. 2, pp. 27-33, 2006.

[7] Andritsos, N., Yiantsios, S. G., and Karabelas, A. J., Cal- cium Phosphate Scale Formation From Simulated Milk Ultrafiltrate Solutions, Food and Bioproducts Processing, vol. 80, no. C4, pp. 223-230, 2002.

[8] Mekmene, O., Leconte, N., Rouillon, T., Quillard, S., Bouler, J. M., and Gaucheron, F., Physicochemical Char- acterisation of Calcium Phosphates Prepared From Milk Ultrafiltrates: Effect of the Mineral Composition, Inter- national Journal of Dairy Technology, vol. 65, no. 3, pp. 334-341, 2012.

[9] Santos, O., Nylander, T., Paulsson, M., and Tragardh, C., Whey Protein Adsorption Onto Steel Surfaces-Effect of Temperature, Flow Rate, Residence Time and Aggrega- tion, Journal of Food Engineering, vol. 74, no. 4, pp. 468-483, 2006.

[10] Yeap, B. L., Wilson, D. I., Polley, G. T., and Pugh, S. J., Retrofitting Crude Oil Refinery Heat Exchanger Networks to Minimize Fouling While Maximizing Heat Recovery, Heat Transfer Engineering, vol. 26, no. 1, pp. 23-34, 2005.

[11] Rosmaninho, R., and Melo, L. F., Calcium Phosphate De- position From Simulated Milk Ultrafiltrate on Different Stainless Steel-Based Surfaces, International Dairy Jour- nal, vol. 16, no. 1, pp. 81-87, 2006.

[12] Rosmaninho, R., Rizzo, G., Muller-Steinhagen, H., and Melo, L. F., Deposition From a Milk Mineral Solution on Novel Heat Transfer Surfaces Under Turbulent Flow Conditions, Journal of Food Engineering, vol. 85, no. 1, pp. 29-41, 2008.

[13] Afgan, N. H., and Carvalho, M. G., Knowledge-Based Expert System for Fouling Assessment of Industrial Heat Exchangers, Applied Thermal Engineering, vol. 16, no. 3, pp. 203208, 1996.

[14] Janknecht, P., and Melo, L. F., Online Biofilm Monitoring, Reviews in Environmental Science and Biotechnology, vol. 2, no. 2, pp. 269-283, 2003.

[15] Pereira, A., and Melo, L. F., Monitoring of Biofilms in the Food and Beverage Industries, in Biofilms in the Food and Beverage Industries, ed. P. M. Fratamico, B. A. Annous, and N. W. Gunther, CRC Press, Woodhead Publishing Lim- ited, Oxford, UK, pp. 131-151, 2009.

[16] Visser, J., and Jeurnink, T. J. M., Fouling of Heat Exchang- ers in the Dairy Industry, Experimental Thermal and Fluid Science, vol. 14, no. 4, pp. 407-424, 1997.

[17] Pereira, A., Mendes, J., and Melo, L. F., Using Nanovibra- tions to Monitor Biofouling, 
Biotechnology and Bioengi- neering, vol. 99, no. 6, pp. 1407-1415, 2008.

[18] Pereira, A., Rosmaninho, R., Mendes, J., and Melo, L. F., Monitoring Deposit Build-Up Using a Novel Mechatronic Surface Sensor (MSS), Food and Bioproducts Processing, vol. 84, no. C4, pp. 366-370, 2006.

[19] Pereira, A., Mendes, J., and Melo, L. F., Monitoring Cleaning-in-Place of Shampoo Films Using Nanovibra- tion Technology, Sensors and Actuators B-Chemical, vol. 136, no. 2, pp. 376382,2009 .

[20] Jenness, R., and Koops, J., Preparation and Properties of a Salt Solution Which Simulates Milk Ultrafiltrate, Nether- lands Milk and Dairy Journal, vol. 16, no. 3, pp. 153-164, 1962.

[21] Santos, O., Nylander, T., Schillen, K., Paulsson, M., and Tragardh, C., Effect of Surface and Bulk Solution Proper- ties on the Adsorption of Whey Protein Onto Steel Surfaces at High Temperature, Journal of Food Engineering, vol. 73, no. 2, pp. 174-189, 2006.

[22] Brito, A. G., and Melo, L. F., Mass Transfer Coefficients Within Anaerobic Biofilms: Effects of External Liquid Ve-locity, Water Research, vol. 33, no. 17, pp. 3673-3678, 1999.

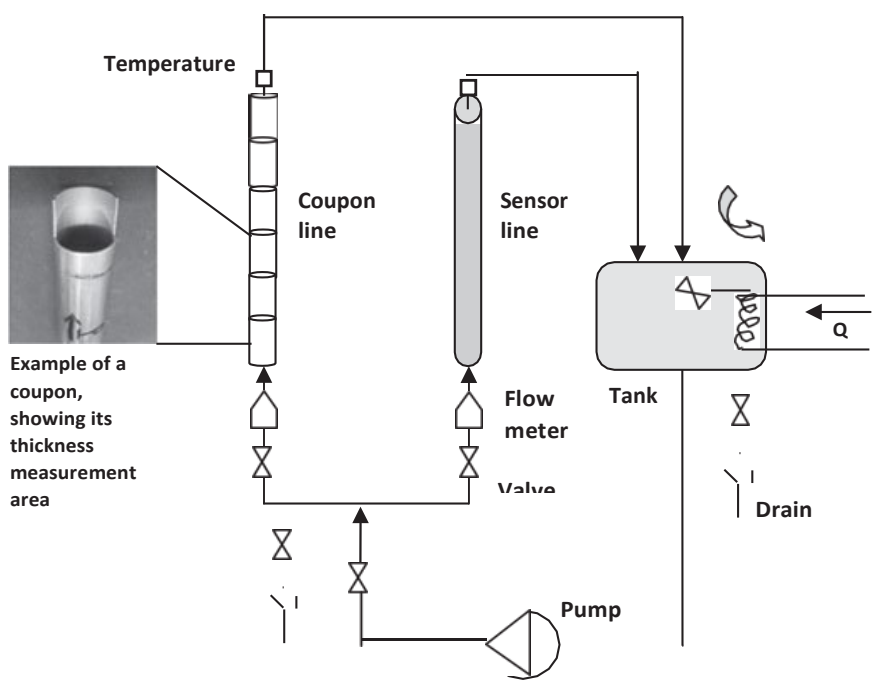

Figure 1 Diagram of experimental rig. 




Figure 2 Normalized amplitude (V) over time ( $\mathrm{min}$ ) for three deposit types at $60^{\circ} \mathrm{C}$ : SMUF, WPI, and SMUF + WPI. (Color figure available online.)

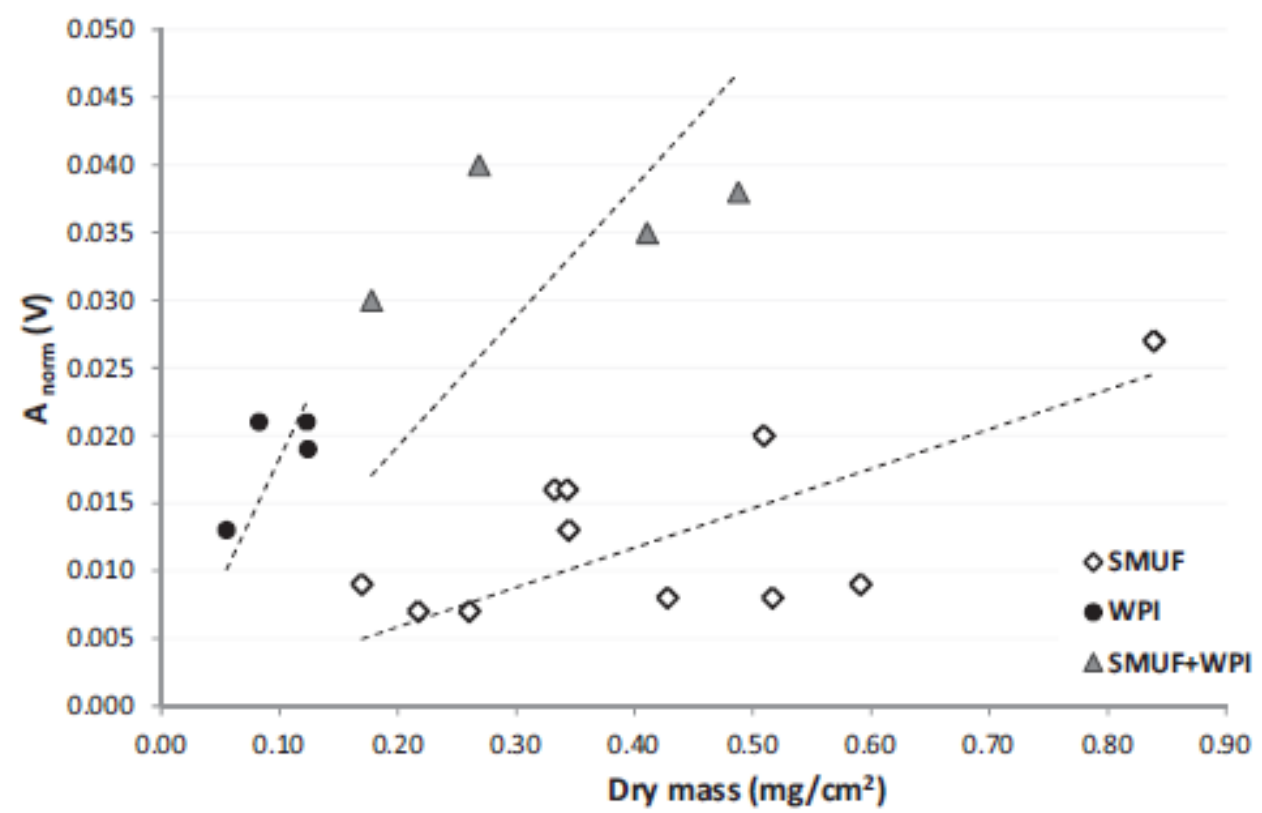

Figure 3 Relationship of normalized amplitude (V) with dry mass $\left(\mathrm{mg} / \mathrm{cm}^{2}\right)$ for three deposit types: SMUF, WPI, and SMUF mixed with WPI. 


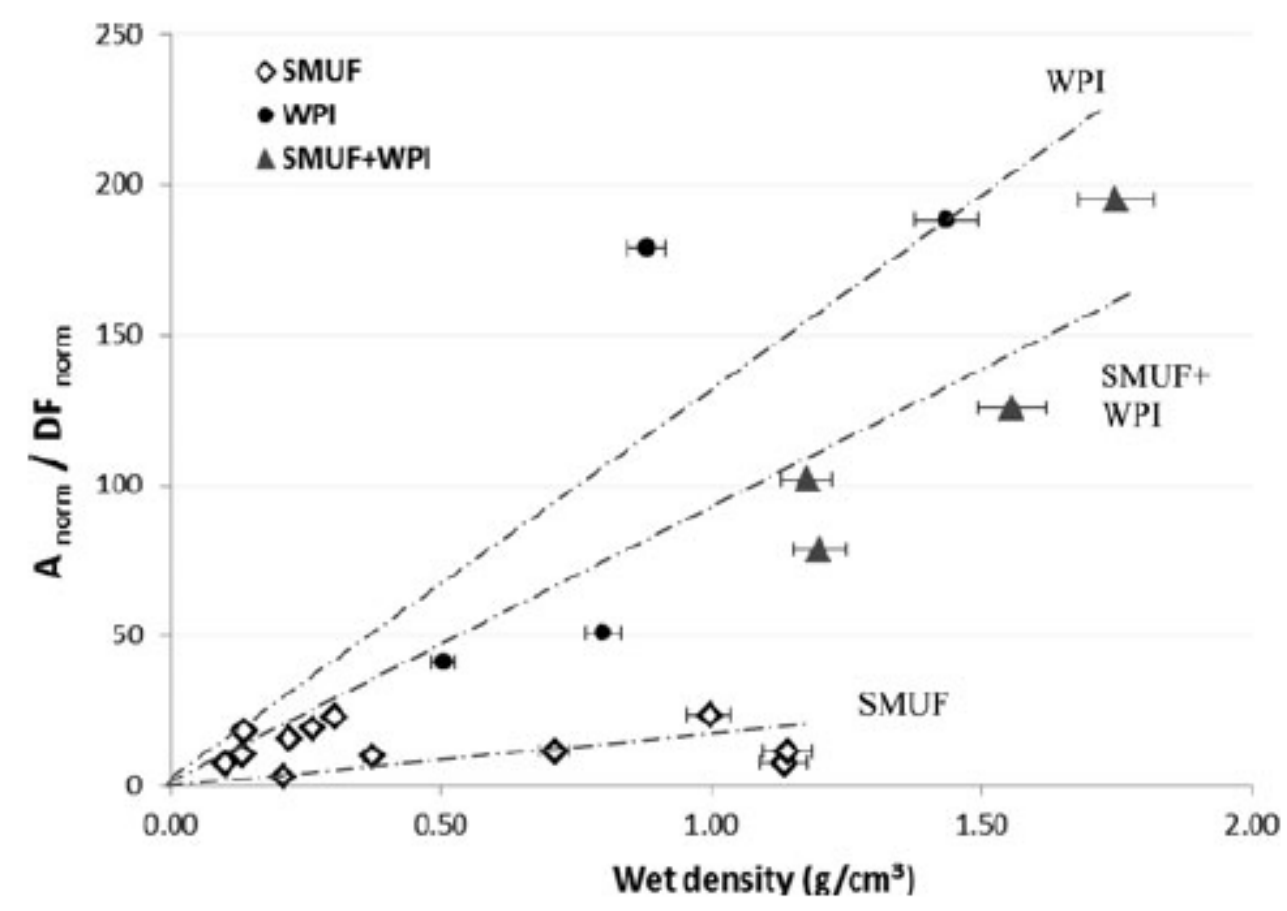

Figure 4 Normalized amplitude-normalized damping factor ratio as a function of wet density $\left(\mathrm{g} / \mathrm{cm}^{3}\right)$ for three types of deposits: SMUF, WPI, and SMUF + WPI. 




(a)

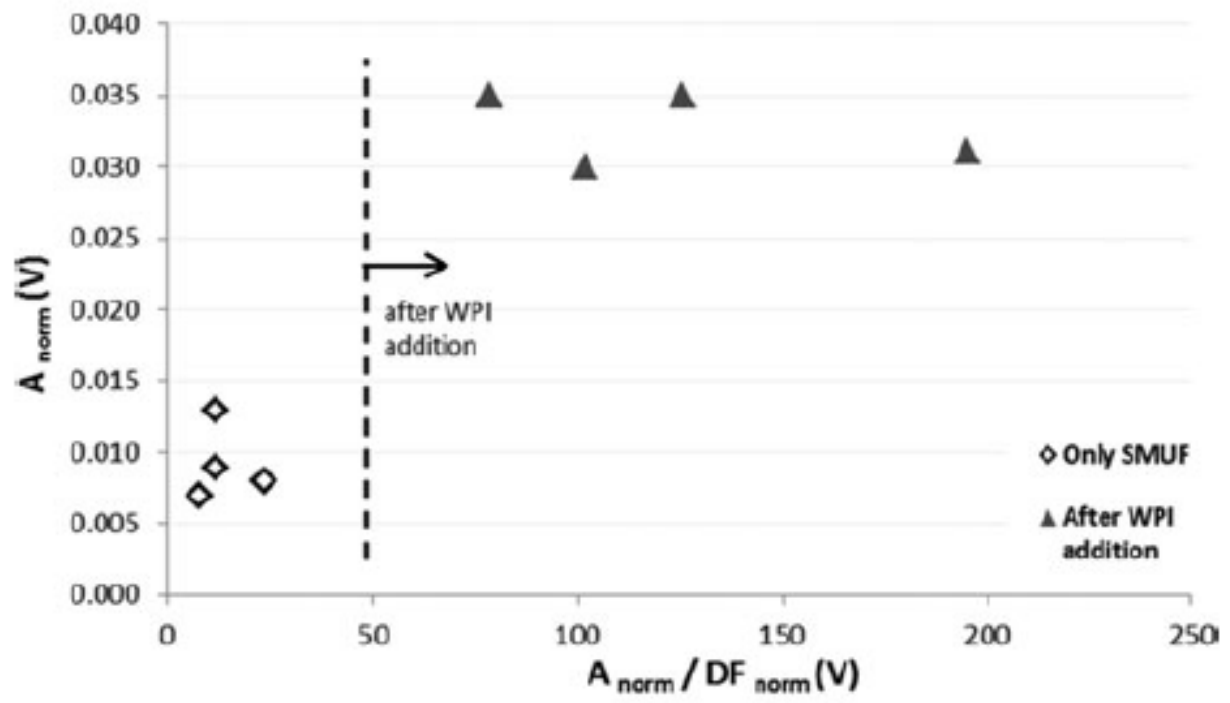

(b)

Figure 5 (a) normalized amplitude related to the normalized amplitude-normalized damping factor ratio for three types of deposits: SMUF, WPI, and SMUF+WPI. (b) Normalized amplitude related to the normalized amplitude-normalized damping factor ratio for the specific case of the SMUF WPI assays. Points show when only SMUF was present and then after WPI addition. 